(C) IEEE

Marti, Enrique, Miguel Angel de Miguel, Fernando Garc ia, and Joshue Perez. "A Review of Sensor Technologie s for Perception in Automated Driving." IEEE Intellig ent Transportation Systems Magazine 11, no. 4 (2019): 94-108. doi:10.1109/mits.2019.2907630. 


\title{
A Review of Sensor Technologies for Perception in Automated Driving
}

\author{
Enrique Martí and Joshué Pérez, Member, IEEE \\ Automated Driving group, Fundación Tecnalia, Derio, 48160 Spain. \\ E-mail: enrique.marti@tecnalia.com \\ Miguel Ángel de Miguel and Fernando García, Member, IEEE
Universidad Carloss III de Madrid, Leganés, 28911, Spain.
}

Abstract-After more than 20 years of research, ADAS are common in modern vehicles available in the market. Automated Driving systems, still in research phase and limited in their capabilities, are starting early commercial tests in public roads. These systems rely on the information provided by on-board sensors, which allow to describe the state of the vehicle, its environment and other actors. Selection and arrangement of sensors represent a key factor in the design of the system. This survey reviews existing, novel and upcoming sensor technologies, applied to common perception tasks for ADAS and Automated Driving. They are put in context making a historical review of the most relevant demonstrations on Automated Driving, focused on their sensing setup. Finally, the article presents a snapshot of the future challenges for sensing technologies and perception, finishing with an overview of the commercial initiatives and manufacturers alliances that will show the intention of the market in sensors technologies for Automated Vehicles. 


\section{Introduction}

E very year more than one million people die on road accidents and several million more get injured [1]. In addition to the social cost, it also has an important economic impact for nations worldwide. According to [2] the most frequent causes for car accidents in the European Union are human related: speeding, driving under the effects of alcohol or drugs, reckless driving, distractions or just plain misjudgments.

Automated Driving systems aim to take the human driver out of the equation. This makes them a tool with the potential to reduce the number of traffic accidents. Based on recent developments and demonstrations around the world, there is a tendency to think that Automated Driving with a high level of automation will be available in a few years. This raises questions about its safety.

The architecture of Automated Vehicles is usually divided into three categories: perception of the environment, behavior planning and motion execution [3]. Automated vehicles obtain information about their surroundings using different sensors, such as cameras, LiDARs and radars. Raw data is processed to extract relevant features which are the input to the following stages (behavior planning and motion execution), that will perform tasks such as path planning, collision avoidance or control of the vehicle among others.

Perception is a very challenging problem for several reasons. First, the environment is complex and highly dynamic, with some cases involving a large number of participants (dense traffic, populated cities). Second, it needs to work reliably under a wide range of external conditions, including lighting and weather (rain, fog, snow, dust). Perception errors are propagated and can be the cause of severe accidents. Some real examples include the 2016 Tesla AutoPilot accident [4], where a man was killed after its car crashed a truck: the camera failed to detect the gray truck against a bright sky while radar detection was discarded as background noise by perception algorithms. Later in 2018, a Tesla model X crashed a highway divider after the lane following system failed to detect faded lines and the concrete divider was not recognized, killing the driver [5]. Also in 2018, an experimental Uber vehicle killed a woman that was crossing the road [6] in the night, dressed in dark clothes. Only the LiDAR provided a positive detection, that was discarded as a false positive by perception algorithms.

Sensor technologies have been surveyed previously in the literature, but usually centered on ADAS implementation $[7,8]$ or at a general level within Automated Driving [9]. One of the main contributions of this work is its focus on the relation between sensors and perception, which provide an integral view of the process that leads from raw sensor data to meaningful information for the driving task.

The content of the article is organized as follows. Section II reviews the sensor technologies commonly used for perception, its drawbacks and advantages, and related emerging technologies that can be used in the future. Section III starts describing the most important competences in perception, to proceed with a state of the art of perception algorithms and techniques grouped by competences. Sensors used on each work are enumerated, and their advantages and disadvantages are discussed. Section IV gives a perspective of the evolution of perception in Automated Driving, presenting the most relevant works and demos in the history of the discipline with a focus in sensor technologies used for each one. Finally, section V contains a discussion of the current state of the discipline and the future challenges for sensors and perception in Automated Driving systems. It includes a review of the most relevant alliances between OEMs (Original Equipment Manufacturers) and technological companies involved in Automated Driving projects at the time of writing the article.

\section{Sensors and Technologies}

This work is focused in exteroceptive sensors, leaving proprioceptive sensors and communications out of the scope of the review. Exteroception in Automated Driving is related with information in the surroundings of the vehicle, as opposed to proprioception that is related with the state of the vehicle itself (speed, accelerations, component integrity).

Next subsections present the advantages, drawbacks and current challenges for the three principal sensor technologies for exteroceptive perception in Automated Driving: artificial vision, radar and LiDAR. Each one is followed with a review of relevant emergent technologies in the field.

After that, a taxonomy of information domains is presented. It is useful for several purposes. First it allows to link sensors technologies with perception algorithms described in section III, since the first provide the raw data needed by the second. Second, the categorization is used to structure a subsequent analysis about the suitability and adequacy of the presented sensing technologies for perception in Automated Driving. This last part includes also the expected performance under different environmental and weather conditions.

\section{A. Artificial Vision}

Artificial vision is a popular technology that has been used for decades in disciplines as mobile robotics, surveillance or industrial inspection. This technology offers interesting features, as the low cost of sensors -for most popular typesand providing range of information types including spatial (shape, size, distances), dynamic (motion of objects by analyzing their displacement between consecutive frames) and semantic (shape analysis).

Cameras available in the market offer a wide range of configurations in resolution (from less than 0.25 to more 
than $40 \mathrm{Mpx}$ ), frame rate (up to thousands of frames per second (FPS)), sensor size, and optics parameters. However, Automated Driving poses some particular challenges to camera sensors and artificial vision technology:

Varying light and visibility conditions. Driving happens at day, at night, indoors, or at dusk or dawn with the sun close to the horizon. Dark spots, shadows, glares, reflections and other effects complicate the implementation of reliable artificial visible algorithms. Extending the capturing spectrum can solve some of these problems. Far infrared (FIR) cameras (wavelength 900-1400 nm) are effective for pedestrian and animal detection [10, 11], in the dark and through dust and smoke. Near Infrared (NIR) (750-900 nm) complements visible spectrum with a better contrast in high dynamic range scenes, and better night visibility. In [12] authors compare visible light, NIR and FIR cameras under different light and atmospheric conditions.

Scenes with a High Dynamic Range (HDR) contain dark and strongly illuminated areas in the same frame, as entering or exiting a tunnel. Common sensor technologies have single shot dynamic range of 60-75 $\mathrm{dB}$, which cause a loss of information in the extremes (under- or overexposure). In 2017 Sony launched a $120 \mathrm{~dB}$ automotive sensor and $2 \mathrm{k}$ resolution. An automotive grade sensor combining HDR capabilities and NIR light detection is analyzed in [13] and the work [14] presents a sensor with 130/170 dB range (global/rolling shutter configurations).

A more extensive review of camera and sensor problems can be found in [15], from the perspective of recording scenes in sports.

\section{1) 3D Technology}

Traditional camera technology is essentially 2D, but there are some types of vision sensors that can perceive depth information. This section describes the three principal types that are already available as commercial devices, although not always targeting the automotive market.

Stereo vision. Depth is calculated [16] from the apparent displacement of visual features in the images captured by two carefully calibrated monocular cameras pointing in the same direction and separated by some distance (known as baseline).

One of the greatest advantages of stereo vision systems is their capability to provide dense depth maps, as opposed to sparse sensors (e.g. LiDARs). Stereo vision drawbacks include issues with low-textured patterns (e.g. solid colors) that difficult establishing correspondences between frames.

Monocular SLAM (Simultaneous Location And Mapping) algorithms share some of the working principles of stereo system: the motion of a single monocular camera creates an artificial baseline between consecutive frames, from which depth and camera motion are estimated. Some works as $[17,18]$ represent a good alternative to stereo sensors for location and mapping.
Structured light. A monocular camera coupled with a device that illuminates the scene with a known pattern of infrared light. Irregular surfaces produce an apparent distortion of the light pattern, that is captured by the camera and translated to a depth map.

Structured light devices overcome some limitations of stereoscopic systems: they do not depend on textured surfaces and have a lower computational cost. However, they require the same high-accuracy calibration [19] and its operative range (usually below 20 meters) is limited by the power of the emitter and the intensity of ambient light. Reflections can affect its performance.

Time-of-flight. Is an active sensing technology [20] based in the same round-trip-time principle of LiDAR sensors (see II C): an emitter composed of infrared LEDs floods the scene with modulated light that is captured by the sensor after being reflected by elements in the environment. The round-trip-time can be calculated for each pixel based on the phase shift of incoming light, which is then translated to a distance.

Using a non-directed source of light (as opposed to the low divergence laser emitter in LiDAR) has advantages as the ability to create dense depth maps and a high refresh rate exceeding $50 \mathrm{~Hz}$. However, its operative range is short for automotive applications (10-20 meters) and has problems working under intense ambient light. Some research lines as indirect time-of-flight [21], pulsed light time-offlight or avalanche photodiodes [22] could increase working range to 50-250 meters.

\section{2) Emerging Vision Technologies}

In event-based vision the elements of the sensor (pixels) are triggered asynchronously and independently when they detect a change on light intensity (an event). The sensor produce a stream of events that can be grouped in time windows for getting a frame-like image. Independence of sensor elements raises the dynamic range of the sensor to $120 \mathrm{~dB}$, allowing high speed applications in low light conditions. [23] shows tracking at 1000 FPS under regular indoor lightning conditions, although the sensor works in sub-microsecond time scales. Events can be the input to visual odometry [24] and SLAM [25] applications, relieving the CPU of time consuming operations on raw images.

There is an active line of research [26] around sensors capturing light polarization, which perform consistently under adverse meteorological conditions and provide exotic types of information (e.g. materials, composition, water in the road).

\section{B. Radar}

Radar technology use high frequency electromagnetic waves to measure the distance to objects based on the round-trip time principle, which is the time it takes the wave to reach the object, bounce on it and travel back to the sensor. 
Most modern automotive radars are based on the Frequency-Modulated Continuous Wave (FMCW) technology, and use digital beamforming [27] to control the direction of the emitted wave. FMCW consists on emitting a signal with a well known and stable frequency that is modulated with another continuous signal that varies its frequency up and down (typically using a triangular shape). Distance is determined using the frequency shift between the emitted and reflected signals. Radars also exploit Doppler effect to get a direct observation of the relative speed of the target with respect to the sensor.

One of the strongest arguments for including radar sensing in automated vehicles is its independence of light and weather conditions. It works in the dark, and detections are almost equally good with snow, rain, fog or dust [28]. Long range radars can see up to $250 \mathrm{~m}$ in very adverse conditions, where no other sensor works.

Radar sensors present some difficulties and drawbacks:

Sensible to target reflectivity. Processing radar data is a tricky task, due in part to the heterogeneous reflectivity of the different materials. Metals amplify radar signal, easing detection of vehicles but increasing the apparent size of small objects as discarded cans in the road, while other materials (e.g. wood) are virtually transparent. This can cause false positives (detect a non existing obstacle) and false negatives (not detecting an actual obstacle).

Resolution and accuracy. Radars are very accurate measuring distance and speed along the line that connects the sensor with a target. However, horizontal resolution depends on the characteristics of the emitted beam. Raw angular resolution in digital beamforming systems falls between 2 to 5 degrees [29], that can be improved to 0.1-1 degrees using advanced processing techniques [30]. With this angular resolution, it can be difficult to separate (detect as independent targets) a pedestrian from a nearby car at $30 \mathrm{~m}$ distance. At $100 \mathrm{~m}$ distance it can be impossible to separate vehicles in neighbor lanes, determine if a vehicle is in our same lane, and even if a detection is a vehicle or a bridge over the road.

\section{1) Emerging Radar Technologies}

One of the most active research area is related with high resolution radar imaging for automobiles. Apart from benefits in target tracking and object separation, a higher resolution can get richer semantic information and enable further applications as target classification and environment mapping. An example can be found in [28], where a $90 \mathrm{GHz}$ rotating radar in the roof of a car is used to map the environment, including vehicles, static objects and ground. The paper [31] demonstrates the feasibility of radars operating between 100 and $300 \mathrm{GHz}$, analyzing atmospheric absorption and reflectivity of materials usually found in driving scenarios.

One of the key technologies that can lead to high resolution radar imaging are meta-material based antennas
$[32,33]$ for efficient synthetic aperture radars. Some manufacturers as Metawave are starting to offer products oriented to automotive sector based on the technology.

\section{LiDAR}

LiDAR (Light Detection And Ranging) is an active ranging technology that calculates distance to objects by measuring round-trip time of a laser light pulse. Sensors for robotic and automotive applications use a low power NIR laser (900-1050 nm) that is invisible and eye-safe. Laser beams have a low divergence for reducing power decay with distance, allowing to measure distances up to $200 \mathrm{~m}$ under direct sunlight. Typically, a rotating mirror is used to change the direction of the laser pulse, reaching $360 \mathrm{de}-$ gree horizontal coverage. Commercial solutions use an array of emitters to produce several vertical layers (between 4 and 128). This generates a 3D point cloud representing the environment. LiDAR sensors are a good choice for creating accurate digital maps, because of their high accuracy measuring distances which averages a few millimeters error in most cases and degrading to 0.1-0.5 meters in the worse [34]. However, they have several drawbacks to take into account.

Low vertical resolution. In low cost models, which usually feature less than 16 layers, vertical resolution (separation between consecutive layers) falls down to 2 degrees. At $100 \mathrm{~m}$ distance, this is translated into a vertical distance of $1.7 \mathrm{~m}$. High end models reduce this to 0.2-0.4 degrees, but at a much higher cost.

Sparse measures (not dense). Commercial device Velodyne HDL64 has a 2 mrad divergence [35] (0.11 degrees) and a vertical resolution of 0.42 degrees. At 50 meters distance, the 0.3 degree gap between layers is equivalent to a blind strip 0.26 meters tall. In low end devices (Velodyne VLP16) this gap grows to 1.5 meters. Small targets can remain undetected, and structures based on wires and bars are virtually invisible.

Poor detection of dark and specular objects. Black cars can appear as invisible to the LiDAR, since they combine a color that absorbs most radiation with a non-Lambertian material that does not scatter radiation back to receiver.

Affected by weather conditions. NIR laser beams are affected by rain and fog because water droplets scatter the light [36], reducing its operative range and producing false measures in the front of the cloud. The effect of dust has been explored in [37]. LiDAR performance in these scenarios is worse than radar, but still better than cameras and human eye.

\section{1) Emerging LiDAR Technologies}

FMCW LiDAR [38] emits light continuously to measure objects speed based on Doppler effect. In the last years some research prototypes suitable for the automotive market start appearing [39]. Apart from improving target tracking 
capabilities, observation of speed can be useful to enhance activity recognition and behavior prediction, for example by detecting the different speeds of limbs and body in cyclists and pedestrians.

Solid state LiDAR is an umbrella term that includes several technologies, two of which are oscillating micromirrors and Optical Phased Array (OPA). The first technology directs laser beams using micro-mirrors that can rotate around two axes. Manufacturer LeddarTech commercializes devices based on this technology [40]. Optical phased arrays [41] is a technology similar to that used for EBF radars that allows to control the direction of the beam with high accuracy and speed. Quanergy [42] is one of the few manufacturers commercializing devices based on this technology.

OPA technology can apply random-access scan patterns over the entire FoV (Field of View). This allows observing only specific regions of interest, and change beam density (resolution) dynamically. These features can be combined to do fast inspection of the full FoV with low resolution, and then tracking objects of interest with a higher resolution for enhanced shape recognition even at far distances.

\section{Relevant Information Domains}

The task of a perception system is to bridge the gap between sensors providing data and decision algorithms requiring information. A classical differentiation between both terms is the following: data is composed by

\section{Table 1. Information taxonomy in Automated Driving domain}

\begin{tabular}{|c|c|c|}
\hline Category & $\#$ & Information type \\
\hline \multirow[t]{2}{*}{ Ego-vehicle } & 1 & Kinematic/dynamic (includes position) \\
\hline & 2 & Proprioceptive (components health/status) \\
\hline \multirow[t]{3}{*}{ Occupants } & 3 & Driver awareness/capacities \\
\hline & 4 & ${ }^{*}$ Driver intentions (mind model) \\
\hline & 5 & Passenger status (needs, risk factors) \\
\hline \multirow[t]{4}{*}{ Environment } & 6 & Spatial features: location, size, shape, fine features \\
\hline & 7 & Identification: class, type, identity \\
\hline & 8 & Semantic features: signs, road marks, regulation \\
\hline & 9 & $\begin{array}{l}\text { Contextual factors: weather, driving situation (e.g. } \\
\text { jam, off-road, emergency) }\end{array}$ \\
\hline \multirow{5}{*}{$\begin{array}{l}\text { External } \\
\text { actors }\end{array}$} & 10 & Spatial features: location, size, shape, fine features \\
\hline & 11 & Kinematic/dynamic: position, motion \\
\hline & 12 & Identification: class, type, identity \\
\hline & 13 & $\begin{array}{l}\text { Semantic features: vehicle lights, pedestrian clothes, } \\
\text { gestures }\end{array}$ \\
\hline & 14 & $\begin{array}{l}\text { * Situational engagement: collaborative/aware } \\
\text { (adults, other vehicles) vs non-collaborative/ } \\
\text { unaware (animals, children) }\end{array}$ \\
\hline
\end{tabular}

raw, unorganized facts that need to be processed, while information is the name given to data that has been processed, organized, structured and presented in a proper context.

Table 1 presents a taxonomy tightly related with the goals of perception stage (section III). It allows to present conclusions about the suitability of sensor technologies for different perception tasks in a clear and organized way. Elements marked with an asterisk are derived information that can be inferred from sensed data but not directly observed. It is mostly related with internal state of external entities, as the intentions of human beings and animals.

\section{E. Using Sensors for Perception}

Sensor selection and arrangement is one of the most important aspects in the design of a perception system for Automated Vehicles. It has a great impact in its cost, with some setups having several times the price of the rest of the vehicle. This epigraph summarizes two aspects of the uttermost importance: type of information acquired and impact of environmental factors. For an analysis of spatial coverage and range see [43].

The characteristics of a sensing technology determines its suitability for acquiring certain types of information, and restricts its range of operative conditions. Figure 1 relates the principal sensing technologies currently used in the automotive market and Automated Driving initiatives with relevant types of information identified in Table 1. The adequacy of a sensor for acquiring a certain type of information (or equivalently, the expected quality of that type of information when captured by that sensing tech-

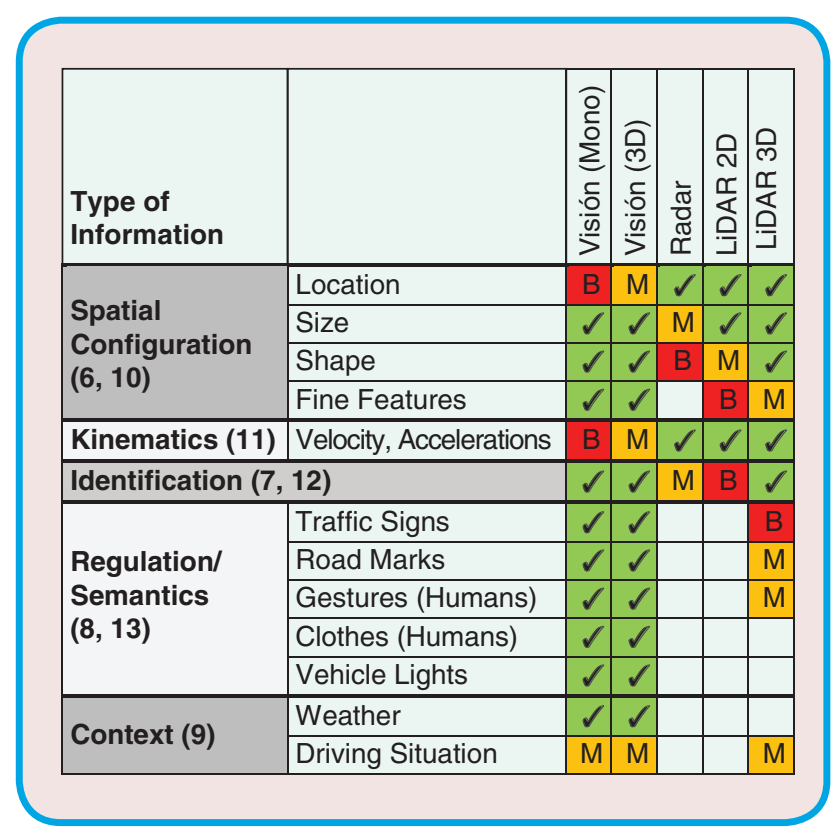

FIG. 1 Sensor adequacy for relevant types of information 
nology) is classified in three levels: Good (green shading, tick), Medium (yellow shading, letter M) and Bad (red shading, letter B).

Sensors and perception are expected to work uninterruptedly during vehicle operation. Weather and other environmental factor can degrade sensor performance, but each technology is affected in a different way. Figure 2 summarizes the effect of common external factors in the performance of the analyzed sensing technologies, using the same notation as Figure 1.

\section{Problems and Applications}

This section analyzes the state of the art in perception systems for Automated Driving. A set of behavioral competences is identified, followed by a systematic literature review that analyzes the solutions for each category, organized by sensor technology.

\section{A. Behavioral Competencies}

Behavioral competencies in Automated Driving "refers to the ability of an Automated Vehicle to operate in the traffic conditions that it will regularly encounter" [44]. The NHTSA defined a set of 28 core competencies for normal driving [45], that have been augmented to a total of 47 by Waymo [46] in their internal tests. Table 2 selects a subset of those behavioral competencies and arranges them in categories that are used to structure the state of the art in perception algorithms in a purpose oriented approach.
This set of competences represents the link between perception and decision (planning), as a counterpart to the information taxonomy presented in the previous section (Table 1), which linked sensors and perception algorithms. Both tables can be combined to evaluate the suitability of sensor technologies for creating some set of Automated Driving capacities.

The next subsections describe the state of the art in perception techniques for the three identified categories of behavioral competencies.

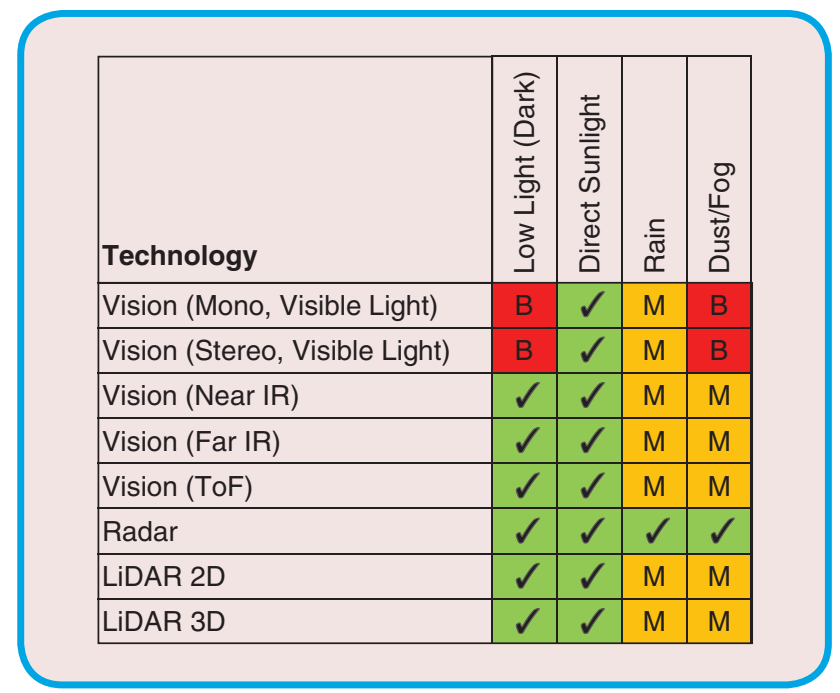

FIG. 2 Sensor robustness under atmospheric and environmental factors

Table 2. Behavioral competences and relation with information taxonomy (see Table 1)

\begin{tabular}{|c|c|c|}
\hline Competence & Information type & Behavior \\
\hline \multirow{4}{*}{$\begin{array}{l}\text { Automatic Traffic Sign Detection and } \\
\text { Recognition (TSDR) }\end{array}$} & 8 & Detect Speed Limit Changes, Speed Advisories, Traffic Signals and Stop/Yield Signs \\
\hline & 8 & Detect Access Restrictions (One-Way, No Turn, Ramps, etc.) \\
\hline & 8 & Detect Temporary Traffic Control Devices \\
\hline & 6,8 & Detect Passing and No Passing Zones \\
\hline \multirow[t]{4}{*}{ Perception of the environment } & 8 & Detect Lines \\
\hline & 6,8 & Detect Detours \\
\hline & 6 & Detect faded/missing roadway markings, signs and other temporary changes in traffic patterns \\
\hline & 9 & Perception under weather or lighting conditions outside vehicle's capability (e.g. rainstorm) \\
\hline \multirow{6}{*}{$\begin{array}{l}\text { Vehicles, pedestrians and other } \\
\text { obstacles detection }\end{array}$} & $10,12,13$ & Detect Non-Collision Safety Situations (e.g. vehicle doors ajar) \\
\hline & $10,11,12,13$ & Detect Stopped Vehicles, Emergency Vehicles, Lead Vehicle, Motorcyclists, School Buses \\
\hline & 6 & Detect Static Obstacles in the Path of the Ego-Vehicle \\
\hline & $6,8,9,10,11,12$ & Detect Pedestrians and Bicyclists at Intersections, Crosswalks and in the Road. \\
\hline & $10,11,12$ & Detect Animals \\
\hline & $10,12,13$ & $\begin{array}{l}\text { Detect instructions from Work Zones and People Directing Traffic in Unplanned or Planned Events, } \\
\text { Police/First Responder Controlling Traffic, Construction Zone Workers Controlling, Citizens } \\
\text { Directing Traffic After a Crash (Overriding or Acting as Traffic Control Device) }\end{array}$ \\
\hline
\end{tabular}




\section{B. Automatic Traffic Sign Detection and Recognition (TSDR)} Traffic signs are visual devices with a well defined aspect, that transmit a clear and precise piece of information about traffic regulation, warnings about factors affecting driving and other informative statements. The spatial and temporal scopes of applicability are also defined in the sign, either explicitly or implicitly. Acquiring information from road traffic signs involves two major tasks: Traffic Sign Detection (TSD) which consists on finding the location, orientation and size of traffic signs in natural scene images, and Traffic Sign Recognition (TDR) or classifying the detected traffic signs into types and categories in order to extract the information that they are providing to drivers [47].

Below are shown the most relevant solutions according to the type of sensor and the technology used.

\section{1) Camera Based Solutions}

Cameras are the most common sensor for TSDR. They can be used for TSR, TSD or both at the same time. As an example of TSR, [48] proposes a method based on the Polar-Fourier Grayscale Descriptor, and [49] a learning method based on a histogram intersection kernel. For TSD, [50] proposes a method based on a fast Convolutional Neural Network $(\mathrm{CNN})$ inspired in the YOLOv2 network. This algorithm can detect the position of the traffic sign and classify it according to its shape. [51] detects stop and yield signs with a statistical template built using color information in different color spaces (YCbCR and ErEgEb). TSD techniques can also be applied to traffic light detection, as in [52], where a Bayesian inference framework to detect and map traffic lights is described. A different approach is proposed by [53] that uses a dual focal camera system composed of a wide angle camera and a telephoto camera which is moved by mirrors in order to get higher quality images of the traffic signs. Camera sensors can also perform TSD and TSR tasks as is shown in the following works where first the signals are detected attending to their color or shape, and then they are classified using machine learning techniques (CNN or SVM) [54-56]. In [57] a system composed by eight roof-mounted cameras which takes images every meter perform offline TDSR to create a database with more than 13,000 traffic signs annotations

\section{2) LiDAR Based Solutions}

LiDAR sensors have been used for TSD. Their 3D perception capabilities are useful to determine the position of the sign and its shape, and can also use the intensity of reflected light to improve detection accuracy based on the high reflectivity of traffic signs. [58] performs detection in three steps: first the point cloud is filtered by laser reflection intensity, then a clustering algorithm is used to detect potential candidates, followed by a filtering step based on the lateral position, elevation and geometry that extracts the signs. [59] goes one step further and makes a primary classification attending to the sign shape (rectangular, triangular and circular).

\section{3) Sensors Fusion Solutions}

A system that combines LiDAR and Cameras can improve the sign detection and recognition as it has the advantages and the information of both sources. [60] trains a SVM with 10 variables: 9 of different color spaces provided by the camera (RGB, HSV, CIEL*a*b*) plus reflection intensity observed by LiDAR. After verifying the 3D geometry of detected signs, a linear SVM classifier is applied to HOG features. [61] method detects traffic signs in LiDAR point clouds using prior knowledge of road width, pole height, and traffic sign reflectance, geometry and size. Traffic sign images are normalized to perform classification based on a supervised Gaussian-Bernoulli deep Boltzmann machine model.

\section{Perception of the Environment}

The purpose of this competence is to characterize and describe the road, which represents the most direct piece of environment of a vehicle. This involves two different aspects: characterize road surface geometry and detect road marks (lanes and complements traffic signs as stops, turns or stopping lines).

Road marks, as traffic signs, are designed to be detected and correctly interpreted by human drivers under a wide variety of external conditions. This is achieved using reflective painting and high contrast colors. Cameras and less frequently LiDARs have been used for detecting them. Road geometry description has been approached using cameras, LiDARs and radars.

In the following lines, the most relevant works about this topic are presented, organized by the type of sensor they use.

\section{1) Camera Based Solutions}

Can be grouped in three categories depending on the specific sensor configuration.

Single Monocular. Using only one camera looking at the road in front of the vehicle it is possible to estimate its shape and lanes, the position of the vehicle in the road and detect road marks. A survey of the most relevant algorithms used for this purpose, mainly for camera sensors is presented in [62].

Multiple Monocular cameras. Some works [63, 64] arrange multiple cameras around the vehicle (typically four, one on each side) to get 360-degree visual coverage of the surroundings. A different configuration is used in [65], where two lateral cameras are used to localize the vehicle.

Binocular or Stereo. The main advantage of binocular cameras is their 3D perception capabilities. It makes possible to detect the ground plane and road boundaries [66, 67], improving road mark detection. 


\section{2) LiDAR Based Solutions}

Main application of LiDARs in road perception is related with detecting the ground plane and road limits [68], as well as detecting obstacles that could occlude parts of the road. In recent works, LiDAR based solutions also take advantage of the higher reflectivity of road marks with respect to the pavement (gray and black material) to detect lane $[69,70]$ and pavement markers [71]. Poor road maintenance can affect markers reflectivity to the point of making them undetectable by LiDAR. This can be solved by fusing LiDAR data with cameras able to perceive non reflective lane marks [63]. Some works use a 2D LiDAR sensor to extract road geometry and road marks [72, 73].

\section{3) Radar Based Solutions}

Radars have been used to determine road geometry based on the principle that the road acts as a mirror for the sensor, returning a very small amount of the emitted power, while the sides of the roads return a slightly higher amount of power. Road limits have been estimated with a maximum error of half a lane at zero distance from the host vehicle and less than one lane width at 50 meters distance. This information can be fused with camera images to improve both detections [74-76].

\section{Detection of Vehicles, Pedestrians and Other Obstacles}

This competence involves moving elements that can be in the path of the vehicle, so it requires extracting more information. Apart from detection and classification, it is also important to determine the position of obstacles with respect to the vehicle, their motion direction, speed, and future intentions when possible. This information will be the input to other systems like path planners or collision avoidance systems (reviewed in [77]).

\section{1) Camera Based Solutions}

Different configurations have been used for camera based obstacle detection, including single monocular camera, multiple cameras, stereo cameras and infrared cameras.

Cameras can be placed in different locations. The front of the vehicle is the most common placement since the most critical obstacles will be in front of the vehicle, but many works explored other positions in order to increase the FoV. A camera placed on the side-view mirror, in the passengers window [78] or looking backwards [79] can prevent backing crash and improve the decision of lane change maneuvers [80-82]. An omnidirectional camera mounted on the top of the vehicle has been used in [83] to detect obstacles and estimate ego-motion.

Stereo cameras are widely used for obstacle detection as they provide 3D information of the position of the obstacles. A large review of the different algorithms used for this kind of cameras can be found in [84]. FIR cameras are independent of scene illumination and can spot obstacles at night [85]. Relevant moving elements (vehicles, pedestrians, animals) are usually hot and, thus, easy to detect with FIR cameras. However, this sensor has to be complemented with other technologies as in [86], since cold obstacles like parked vehicles or trees can be not perceived. [87] presents and explains in detail several camera solutions and the algorithms used for detection.

\section{2) LiDAR Based Solutions}

LiDAR technology allows to detect and classify surrounding elements, providing a very accurate $3 \mathrm{D}$ position and its shape. As it is an active sensor its performance is not affected by the illumination of the scene, so it can work also at night. Several approaches for LiDAR obstacle detection are shown in [88].

\section{3) Radar Based Solutions}

The primary use of automotive radars is detection and tracking of other vehicles on the road, thanks to their high accuracy measuring target distances and relative speed, long range detection and performance in adverse weather conditions [89]. Radars have low angular resolution, causing misplacement of detected elements and reporting targets that are close to each other as a single larger object. A common solution consists on fusing radar detections with other sensors as cameras [90] or LiDARs [91].

\section{4) Multiple Sensors Fusion Solutions}

This competence requires estimating a large number of variables simultaneously, creating difficulties for any single sensor solution. This is a good scenario for sensor fusion systems, that can combine the strengths of each sensor to improve the solution.

Radar and LiDAR fusion [91] increases the precision of the speed obtained only with LiDAR and keeps a good position and speed estimation quality when radar is unavailable (especially in curvy roads). Radar and vision fusion techniques use radar information to locate areas of interest on the images, which are then processed to detect vehicles and improve their position estimation [92]. LiDAR and vision sensors are fused in [93, 94]. Obstacles are detected and tracked with the LiDAR, and the targets are classified using a combination of camera and LiDAR detections.

\section{Relevant Works and Demos}

This section describes some of the most relevant technological demonstrations, competitions, challenges and commercial platforms related with Automated Driving, starting from pioneering works in late 1980s until present day. Figure 3 arranges them in a timeline, with the focus on the sensors equipped by each platform.

The timeline allows to discern different stages ("ages") in the development of Automated Driving technology, and 


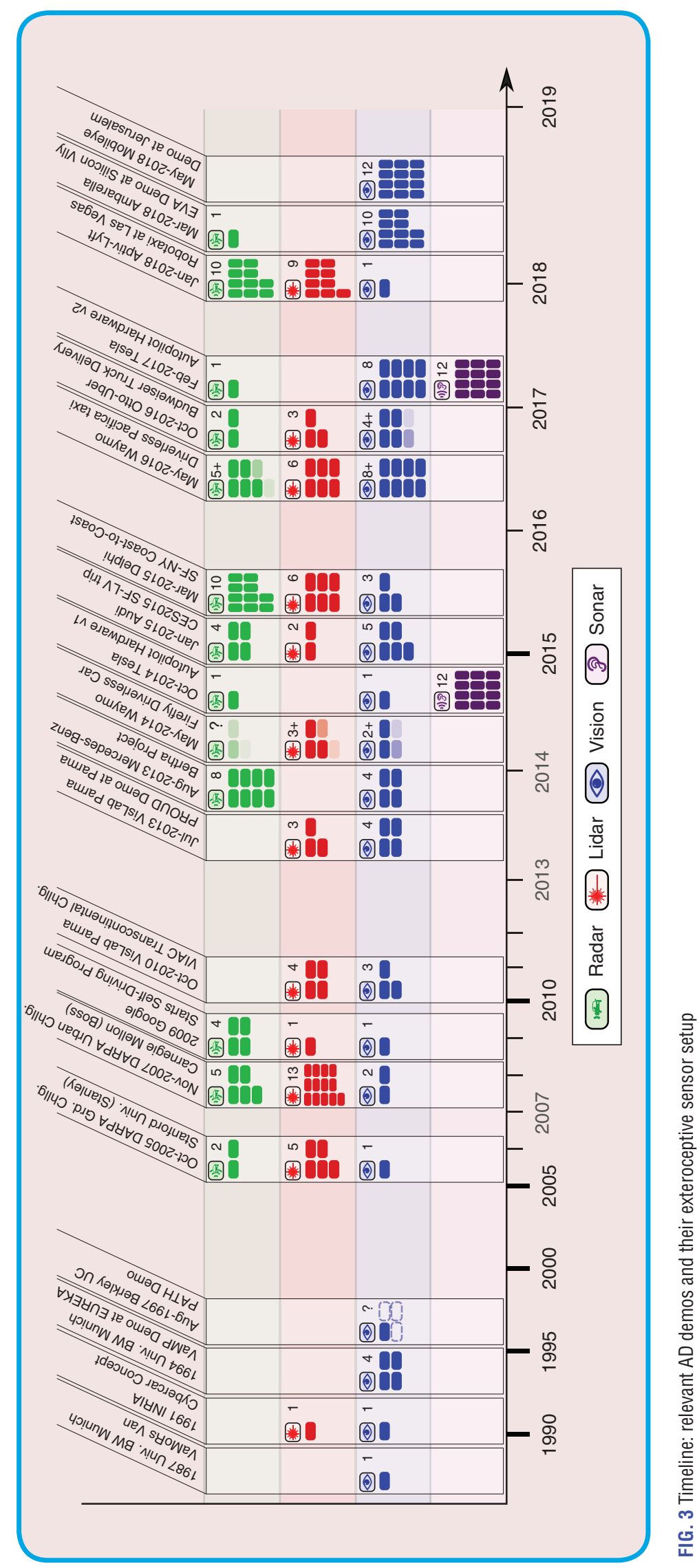

to identify trends and approaches from the perception point of view for Automated Vehicles.

\section{A. Pioneer Works (1980-2000)}

Pioneer works in Automated Driving starts around mid-1980s focused in vision based techniques, which represented a huge computational burden for the embeddable computers of the time. Automated Vehicles VaMoRs [95] and VaMP [96] from Bundeswehr University of Munich used a saccadic vision system: cameras on a rotating platform that focus in relevant elements. The University of Parma started its project ARGO in 1996. The vehicle completed over $2000 \mathrm{~km}$ of autonomous driving in public roads [97], using a two camera system for road following, platooning and obstacle avoidance.

The Cybercar concept is born in early 1990s [98] as an urban vehicle with no pedals or steering wheel. In 1997 a prototype is installed in Schippol airport to transport passengers between terminal and parking [99]. It used a LiDAR and vision system to drive automatically in a dedicated lane with semaphores and pedestrian crossings.

Also in 1997, the National Automated Highway System Consortium presented a demonstration of Automated Driving functionalities [100], intended to be a proof of technical feasibility. The demo showed road following functionality based on vision sensors, distance maintenance based on LiDAR, vehicle following based on Radar and other functionalities including cooperative maneuvers and mixed environments.

\section{B. Proof of Feasibility (2000-2010)}

In year 2004 DARPA started its Grand Challenge series to foster development of Automated Driving technologies. The achievements over those three years not only represented a huge leap forward, but also called the attention of powerful agents. Two first challenges (2004 and 2005) consisted in covering a route over dirt roads with off-road sections, with a strong focus in navigation and control. Stanford University won the 2005 edition, equipping its vehicle Stanley with 5 LiDAR units, a frontal camera, GPS sensors, an IMU, wheel odometry and two automotive radars [101]. The Urban Challenge (2007) changed the focus to interaction with other vehicles, pedestrians and obeying complex traffic regulations. Carnegie Mellon University team ended in first position with its 
vehicle Boss [102, 103], featuring a perception system composed by two video cameras, 5 radars and 13 LiDAR (including a roof mounted unit of the novel Velodyne 64HDL).

These events triggered the attention of Google. The company hired around 15 scientists from the DARPA challenge, including the winners of 2005 and 2007 [104], [105]. Google's (and Waymo's) approach to self-driving vehicles is largely founded in LiDAR and 3D mapping technologies [106]. All their vehicles have had a roof-mounted spinning LiDAR: Toyota Prius (2009), the Firefly prototype (2014) and Chrysler Pacifica (2016-present).

The University of Parma created the spin-off VisLab in 2009. They are strong supporters of artificial vision as the main component of perception systems for AD. In 2010 they completed the VisLab Intercontinental Autonomous Challenge (VIAC): four automated vans drove from Italy to China over public roads that included degraded dirt roads and unmapped areas [107]. The leading vehicle did perception (with cameras and LiDARs), decision and control, with some human intervention for selecting the route and managing critical situations [108]. In 2013 the PROUD test put a vehicle with no driver behind the wheel in Parma roads for doing urban driving in real traffic [109].

\section{Race to Commercial Products (2010-Present)}

In the last decade the landscape of Automated Driving has been dominated by private initiatives that foresee the coming of Level 4 and 5 systems in a few years. This vision gave birth to several companies devoted to this end, most of which were founded by people coming from the DARPA experience, or hired them to lead the project [106].

Examples include the nuTonomy (co-founded by the leader of the MIT team in 2007 Challenge), Cruise (founded by a member of the same team), Otto (founded by a participant in 2004 and 2005 Challenges), Uber (hired up to 50 people from the CMU Robotics Lab), Zoox robotaxi company (co-founded by a member of the Stanford Autonomous Driving team) [110], and Aurora (similar story with people from Uber, MIT and Waymo [111]).

Car manufacturers reacted a bit slower. Some of them started independent research lines, for example BMW has been testing automation prototypes in roads since 2011 [112] and Mercedes-Benz Bertha project [113] drove in 2013 a $103 \mathrm{~km}$ route in automated mode using close-to-market sensors (8 radars and 3 video cameras), but in the end most manufacturers have created coalitions with technological startups as enumerated in section V B 1.

Mobileye started working in a vision-only approach to Automated Driving a few years ago. After testing in real conditions [114], they presented a demo with an automated Ford equipped just with 12 small monocular cameras for fully Automated Driving in 2018 [115].

Tesla entered the Automated Driving scene in 2014. All their vehicles were equipped with a monocular camera (based on Mobileye system) and an automotive radar that enabled the Level 2-3 AutoPilot functionality. Starting 2017 new Tesla vehicles include the "version 2" hardware, composed by a frontal radar, 12 sonars, and 8 cameras. This sensor set is claimed to be enough for full Level 5 Automated Driving [116], which will be available for a fee (when ready) through a software update.

In 2015 VisLab was acquired by Ambarella, a company working on low power chips able to process high resolution dense disparity maps from stereo cameras [117]. Its latest demo [118] fused data from 10 stereo pairs into an ultrahigh resolution 3D scene delivering 900 million points per second. Long range vision mix a forward facing 4k stereo pair with a radar for better performance under low light or adverse weather conditions.

Delphi Automotive completed in 2015 an automated trip between San Francisco and New York city using a custom Audi Q5 with 10 radars, 6 LiDARs and 3 cameras onboard. In 2017 they acquired nuTonomy (the first company to deliver a robotaxi service in public roads) and created Aptiv. Aptiv presented an automated taxi for CES conference in January 2018, as part of a 20 vehicle fleet that has been serving a set of routes in Las Vegas for some months. The taxis have an extensive set of 10 radars and 9 LiDARs embedded in the bodywork, plus one camera.

Meanwhile, Waymo has grown a fleet of Chrysler Pacifica minivans that has self-driven 10 million miles by October 2018. Their efforts have reportedly cut prices of LiDAR sensors to less than one tenth in a few years. They claim to have created two "new categories of LiDAR" [119] in the way, one for close range perception including below the car, and the other for long range. The long-range LiDAR can reportedly zoom dynamically into objects on the road, letting the vehicle see small objects up to $200 \mathrm{~m}$ away. This reminds the features of OPA solid state LiDARs (see section II C 1): random sampling across the scanning area and adaptive resolution.

\section{Discussion}

The last section of this article presents a discussion of the future challenges for sensors and perception systems in new Automated Vehicles, both from the technical and implantation point of view. A description of the next commercial initiatives and OEMs forecasts is shown followed by the final conclusions.

\section{A. Future Challenges}

Sections II and III show many works that solve the most important perception competences, based on different types of sensors and with a large variety of algorithms. Translating these solutions into a functional, safe and secure commercial Automated Vehicle requires overcoming additional difficulties. 


\section{1) Technical Challenges}

Sensor setups in Automated Driving are usually focused on the areas relevant for the usual driving tasks (covered in section III). But for a commercial system expected to work in the real world there are still some specific challenges that do not have a proper solution yet.

Very short distance, including close to or below the car. A person, animal or object right below the vehicle or intersecting the path of the wheels represents a safety issue. While most situations can be anticipated when the element approaches the vehicle from the distance, it is not the case right before starting the vehicle, while executing high accuracy maneuvers in certain conditions (close to people or other moving elements). This problem can be tackled by adding redundant sensors like [83] which uses a 360-degree-view parking system or a special LiDAR monitoring this area used by Waymo. In the future there will be a need of specific devices for this task.

Very long distance. Detection and classification above 200 meters is an open issue. Among current approaches, Ambarella integrates an Ultra High Resolution camera (cited in IV) that is claimed to be enough for discerning small objects at that target distance, subject to the limitations of visible light cameras. Solutions based on saliency (a common term in artificial vision [120-122] to name relevancy or importance) can be an alternative to the high resolution and computational cost associated to brute force approach- es. Solid state LiDAR capable of random and adaptive sampling is a potential candidate solution for such technology, achieving something similar to Waymo's claims about their custom built LiDARs.

Environmental and weather conditions. Section II summarizes the suitability of common technologies under different conditions, some of which surpass human capacities. This is an always active field of research, following the road when most marks are covered by snow, improving detection under heavy rain or dense fog are examples of problems that can be solved at sensing level without requiring further efforts on processing algorithms.

\section{2) Implantation Challenges}

The final goal of research in automated driving is to bring technologies to market, either for private customers or for shared applications (automated fleets). Commercialization and implantation is feasible only if products fulfill certain scalability, costs, and durability requirements. Some sensors are among the most expensive and fragile components of a vehicle, so their implantation is a key factor in the development of automated driving vehicles.

Production scalability and costs. Mature technologies as visible light cameras and radars have already scaled up their production and reduced costs so that every vehicle can equip them without a significant impact on its price. This remains a challenge for LiDAR devices and other

Table 3. OEM projects and alliances in Automated Driving

\begin{tabular}{|c|c|c|c|c|c|c|}
\hline OEM & Test site & Technologies & Since & Collaborations & Forecast & Test fleet \\
\hline Ford & $\begin{array}{l}\text { Detroit, Arizona \& } \\
\text { California (USA) }\end{array}$ & LiDAR, and mapping & $\sim 2016$ & $\begin{array}{l}\text { Argo, Velodyne, } \\
\text { SAIPS, civilmaps. }\end{array}$ & Level 4 (2021) & $\begin{array}{l}\text { Fusion Hybrid ( } \\
\sim 100 \text { by 2018) }\end{array}$ \\
\hline GM & $\begin{array}{l}\text { Detroit, S. Francisco } \\
\& \text { Scottsdale (USA) }\end{array}$ & LiDAR, HD map, radar, camera & $\sim 2016$ & $\begin{array}{l}\text { Waymo and Jaguar- } \\
\text { Land Rover }\end{array}$ & 2020 (Fortune) & $\sim 50$ vehicles (2017) \\
\hline Renault-Nissan & Japan, USA \& China & $\begin{array}{l}\text { Front radar, LiDAR. Speed/ } \\
\text { steering control }\end{array}$ & $\sim 2017$ & Transdev, Microsoft. & $\begin{array}{l}<2030 \text { (Level 5) } \\
2020 \text { (Level 3) }\end{array}$ & - \\
\hline Daimler & Germany & Vision, data fusion, radar. & 2015 & Bosch & 2020 & $\begin{array}{l}\text { Commercial cars } \\
\text { (Level 2) }\end{array}$ \\
\hline $\begin{array}{l}\text { Volkswagen- } \\
\text { Audi Group }\end{array}$ & Germany & $\begin{array}{l}\text { LiDAR, data fusion, adaptive } \\
\text { cruise control, Trafic Jam } \\
\text { Assist, self-parking }\end{array}$ & 2015 & $\begin{array}{l}\text { Delphi (2015) } \\
\text { Aurora (2017) }\end{array}$ & 2025 (Level 4) & $\begin{array}{l}\text { Commercial cars } \\
\text { (Level 3, Traffic } \\
\text { Jams) }\end{array}$ \\
\hline BMW & Germany, China & Vision, LiDAR, DGPS & 2011 & Intel, Baidu, HERE & 2022 (Level 5) & $\begin{array}{l}\text { Commercial cars } \\
\text { (Level 2) }\end{array}$ \\
\hline Waymo & California (USA) & $\begin{array}{l}\text { LiDAR, vision system, radar, } \\
\text { data fusion, RT Path plan.. }\end{array}$ & 2010 & $\begin{array}{l}\text { Fiat-Chrysler, } \\
\text { Velodyne. }\end{array}$ & - & $\begin{array}{l}100 \text { autonomous } \\
\text { Pacifica minivans }\end{array}$ \\
\hline Volvo & $\begin{array}{l}\text { Sweden. \& Uber: San } \\
\text { Francisco, Pittsburgh }\end{array}$ & Vision, LiDAR, GPS, V2I & 2011 & $\begin{array}{l}\text { Uber (U.S), Autoliv } \\
\text { (Sweden) }\end{array}$ & $\sim 2020$ & $\begin{array}{l}\text { Commercial cars } \\
\text { (Level 2) }\end{array}$ \\
\hline Tesla & USA & Camera, radar, Al & $\sim 2015$ & $\begin{array}{l}\text { Apple, Mobileye and } \\
\text { Nvidia }\end{array}$ & 2020 Level 5) & $\begin{array}{l}\text { Commercial cars } \\
\text { (Level 2) }\end{array}$ \\
\hline Hyundai & South Korea & Al, LiDAR, Camera & 2014 & KIA, Aurora & $\begin{array}{l}\text { AD Level 3. } 2020 \\
\text { (Highways). } 2030 \\
\text { (city streets) }\end{array}$ & - \\
\hline
\end{tabular}


breakthrough technologies. It is difficult to get an exact estimation of an acceptable cost, it depends on the use of the vehicle (private or commercial fleet) in between many other factors. For a discussion on costs and impact of Automated Mobility services, see [123].

Durability and tolerance to failure. The perception system of an Automated Vehicle must work for long periods under harsh conditions, as the rest of critical components in a vehicle. Low mean-time between-failures (as for mechanical LiDARs), external factors (a stone chip at high speeds can damage a sensor) or intentional attacks [124] are important factors to consider in the future sensors technologies.

\section{B. Commercial Initiatives}

In the last decade the automotive market has grown the offer and complexity of ADAS [125]. The most requested ADAS in 2009 [126] were Anti-lock braking system and Parking Assistance by Warning (SAE Level 0). Today most advanced cars equip an ensemble of ADAS that place them between SAE Levels 2 and 3.

\section{1) OEMs in Automated Driving}

Around 2015 most important OEMs decided to take serious initiatives towards bringing high and fully Automated Driving (SAE Levels 4 and 5) to the market. In order to accelerate their roadmaps, they established alliances with technological companies startups and technology/ research centers that can hint about their approach to $\mathrm{Au}-$ tomated Driving.

Table 3 shows a resume of the most promising research and collaboration for Automated Driving with OEMs involved. The most relevant works are leaded by Ford, GM and Daimler. However, the influences of Waymo and Tesla, and the alliances with other actors (NVIDIA, Apple or Intel-Mobileye) plays an important role in this automated race. Another important consideration is that most of the OEMs started their Automated program just two years ago.

\section{Conclusions}

Choosing the sensors configuration of an automated vehicle can be challenging. Each sensor has different strengths and weaknesses regarding the type of information acquired, overall accuracy and quality and working conditions. This survey has reviewed the most popular sensors technologies, describing their characteristics and how they are applied to get useful information to solve the main perception competences. The relevant works and demos section provide a good perspective of how different manufacturers and research groups do perception tasks and which kind of sensors they use for that purpose. Finally, the section V B 1 can form an intuition about how manufacturers are approaching the development of Autonomous Vehicles and how are they planning to get there.

\section{Acknowledgment}

This work has been partly supported by ECSEL Project ENABLE-S3 (with grant agreement number 692455-2), by the Spanish Government through CICYT projects (TRA201563708-R and TRA2016-78886-C3-1-R).

\section{About the Authors}

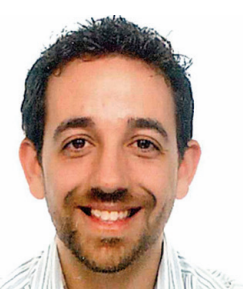

Enrique Martí is a Senior Researcher on Automated Driving at Tecnalia Research and Innovation since 2017. He received his $\mathrm{PhD}$ in Computer Science from University Carlos III de Madrid in 2015. He has more than 10 years of experience in Sensor Fusion and Estimation, including participation in R\&D projects and creation of commercial sensor fusion products for UAV/UGV navigation and surveillance systems. His research interests include sensor technologies, information fusion, machine learning and optimization applied to automated vehicles.

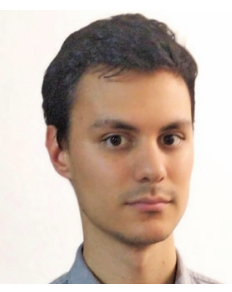

Miguel Ángel de Miguel is a Ph.D. student and an assistant lecturer at University Carlos III de Madrid. He received the B.S degree in electronics engineering in 2015 and the M.S. degree in industrial engineering in 2017, both at University Carlos III de Madrid. In 2013 he joined the Intelligent Systems Lab where he has collaborated in industrial research projects for four years. His research interests include the areas of path planning and control with a focus on applications for autonomous vehicles.

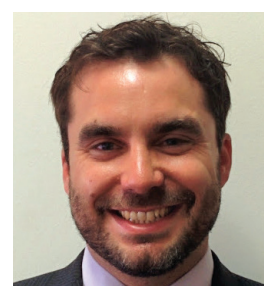

Fernando García is Professor at Universidad Carlos III de Madrid. His research focus in Intelligent Vehicles and Intelligent Transportation Systems involving the use of Computer Vision, Sensor Fusion, and Human Factors. He is member of the BoG and Vice-president of the Spanish Chapter of the IEEE-ITSS since January 2017. He has been recipient of the Barreiros Foundation Award in 2014 and finalist to the best PhD Thesis Dissertation Award in the period 2013-2015 given by ITSS Spanish Chapter.

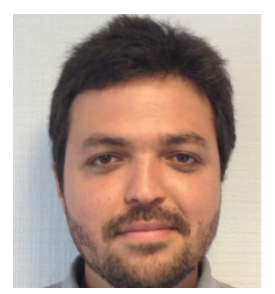

Joshué Pérez is a Research leader on Automated Driving at Tecnalia Research and Innovation, since 2015 . He received the B.E. degree in electronic engineering from the Simon Bolívar University, Venezuela, in 2007. His M.E. degree and his Ph.D. degree from the University Complutense of Madrid were achieved in 
2009 and 2012, respectively. He has more than 11 years of experience in the Intelligent Transportation System field, and more than 100 publications related to Automated Driving and ADAS.

\section{References}

[1] W. H. Organization, Global status report on road safety 2015. World Health Organization, 2015.

[2] P. Thomas, A. Morris, R. Talbot, and H. Fagerlind, "Identifying the causes of road crashes in Europe," Ann. advances automotive medicine. Assoc. Advancement Automotive Medicine. Scientific Conf., vol. 57, pp. 13-22, 2013.

[3] S. Behere and M. Torngren, "A functional architecture for autonomous driving," in Automotive Software Architecture (WASA), 2015 First International Workshop on. IEEE, 2015, pp. 3-10.

[4] NTSB, NTSB Opens Docket on Tesla Crash p. 702, 2017. https://www .ntsb.gov/news/press-releases/Pages/PR20170619.aspx

[5] --, Preliminary Report HWY18FH011 Tech. Rep., 2018. https://www .ntsb.gov/investigations/AccidentReports/Pages/HW Y18FH011 -preliminary.aspx

[6] --, Preliminary Report HWY18MH010 National Transportation Safety Board, Tech. Rep., 2018. https://www.ntsb.gov/investigations/ AccidentReports/Reports/HWY18MH010-prelim.pdf

[7] S. Yenkanchi, "Multi Sensor Data Fusion for Autonomous Vehicles," Ph.D. dissertation, University of Windsor, 2016. https://scholar .uwindsor.ca/etd $/ 5680$

[8] A. Ziebinski, R. Cupek, H. Erdogan, and S. Waechter, "A survey of ADAS technologies for the future perspective of sensor fusion," Lecture Notes Computer Sci. (including subseries Lecture Notes Artificial Intell. Lecture Notes Bioinformatics), vol. 9876 LNCS, pp. 135-146, 2016.

[9] S. Pendleton, H. Andersen, X. Du, X. Shen, M. Meghjani, Y. Eng, D. Rus, and M. Ang, "Perception, Planning, Control, and Coordination for Autonomous Vehicles," Machines, vol. 5, no. 1, pp. 6, feb 2017.

[10] R. O'Malley, M. Glavin, and E. Jones, "A review of automotive infrared pedestrian detection techniques," Signals and Systems Conference, 208.(ISSC 2008). IET Irish, pp. 168-173. 2008.

[11] B. Besbes, A. Rogozan, A. M. Rus, A. Bensrhair, and A. Broggi, "Pedestrian detection in far-infrared daytime images using a hierarchical codebook of surf," Sensors (Switzerland), vol. 15, no. 4, pp. 8570-8594, apr 2015.

[12] N. Pinchon, O. Cassignol, A. Nicolas, F. Bernardin, P. Leduc, J.-P. Tarel, R. Brémond, E. Bercier, and J. Brunet, "All-Weather Vision for Automotive Safety: Which Spectral Band?," in Advanced Microsystems for Automotive Applications 2018. Springer, Cham, sep 2018, pp. 3-15.

[13] S. Maddalena, A. Darmon, R. Diels, "Automotive CMOS image sensors," Advanced Microsystems Automotive, ..., pp. 401-412, 2005.

[14] M. Strobel and D. Döttling, "High dynamic range CMOS (HDRC) imagers for safety systems," Advanced Opt. Technologies, vol. 2, no. 2, pp. 147-157, jan 2013.

[15] B. Pueo, "High speed cameras for motion analysis in sports science," $J$. Human Sport Exercise, vol. 11, no. 1, pp. 53-73, dec 2016.

[16] R. A. Hamzah and H. Ibrahim, "Literature survey on stereo vision disparity map algorithms," J. Sensors, vol. 2016, pp. 1-23, dec 2016.

[17] J. Engel, T. Schöps, and D. Cremers, "LSD-SLAM: Large-Scale Direct monocular SLAM," Lecture Notes Computer Sci. (including subseries Lecture Notes Artificial Intell. Lecture Notes Bioinformatics), vol. 8690 LNCS, NO. PART 2, pp. 834-849, 2014. in Springer, Cham,

[18] J. Engel, V. Koltun, and D. Cremers, "Direct Sparse Odometry," IEEE Trans. Pattern Anal. Mach. Intell., vol. 40, no. 3, pp. 611-625, jul 2018

[19] P. Garbat, W. Skarbek, and M. Tomaszewski, "Structured light camera calibration," Opto-electronics Rev., vol. 21, no. 1, pp. 23-38, jan 2013.

[20] M. Hansard, L. Seungkyu, C. Ouk, and R. Horaud, Time-of-Flight Cameras Principles, Methods and Applications. Springer, 2011.

[21] F. Villa, R. Lussana, D. Bronzi, F. Zappa, and A. Giudice, "3D SPAD camera for Advanced Driver Assistance," 2017 International Conference of Electrical and Electronic Technologies for Automotive, no. November, 2017.

[22] Panasonic, Panasonic Develops Long-range TOF Image Sensor 2018. https://news.panasonic.com/global/press/data/2018/06/en180619-3/ en180619-3.html

[23] E. Mueggler, B. Huber, and D. Scaramuzza, "Event-based, 6-DOF pose tracking for high-speed maneuvers," in IEEE International Conference on Intelligent Robots and Systems. IEEE, sep 2014, pp. 2761-2768.

[24] A. Censi and D. Scaramuzza, "Low-Latency Event-Based Visual Odometry," in IEEE International Conference on Robotics and Automation (ICRA), Hong Kong, 2014, pp. 703-710.

[25] A. R. Vidal, H. Rebecq, T. Horstschaefer, and D. Scaramuzza, "Ultimate SLAM? Combining Events, Images, and IMU for Robust Visual SLAM in HDR and High Speed Scenarios," IEEE Robotics and Automation Letters, vol. 3, no. 2, 2017.

[26] M. Garcia, T. Davis, S. Blair, N. Cui, and V. Gruev, "Bioinspired polarization imager with high dynamic range," Optica, vol. 5, no. 10, pp. 1240 , oct 2018.

[27] J. Hasch, "Driving towards 2020: Automotive radar technology trends," in 2015 IEEE MTT-S International Conference on Microwaves for Intelligent Mobility, ICMIM 2015. IEEE, apr 2015, pp. 1-4.

[28] G. Reina, D. Johnson, and J. Underwood, "Radar sensing for intelligent vehicles in urban environments," Sensors (Switzerland), vol. 15, no. 6 , pp. 14661-14678, jun 2015.

[29] M. Schneider, Automotive Radar - Status and Trends Tech. Rep., 2005. http://citeseerx.ist.psu.edu/viewdoc/download?doi=10.1.1.329.3571 $\{\backslash \&\}$ rep=rep1 $\{\backslash \&\}$ type=pdf

[30] D. Kissinger, "Millimeter-Wave Receiver Concepts for $77 \mathrm{GHz}$ Automotive Radar in Silicon-Germanium Technology, ser," SpringerBriefs in Electrical and Computer Engineering. Boston, MA: Springer US, 2012.

[31] M. Köhler, J. Hasch, H. L. Blöcher, and L. P. Schmidt, "Feasibility of automotive radar at frequencies beyond $100 \mathrm{GHz}$," Int. J. Microwave Wireless Technologies, vol. 5, no. 1, pp. 49-54, feb 2013.

[32] E. Brookner, "Metamaterial Advances for Radar and Communications," in IEEE International Symposium on Phased Array Systems and Technology (PAST). IEEE, oct 2016, pp. 1-9.

[33] T. Sleasman, M. Boyarsky, L. Pulido-Mancera, T. Fromenteze, M. F. Imani, M. S. Reynolds, and D. R. Smith, "Experimental Synthetic Aperture Radar with Dynamic Metasurfaces," IEEE Trans. Antennas Propag., vol. 65, no. 12, pp. 6864-6877, feb 2017.

[34] M. E. Warren, "Automotive lidar technology," in 2019 Symposium on VLSI Circuits. IEEE, 2019, pp. C254-C255.

[35] C. Glennie and D. D. Lichti, "Static calibration and analysis of the velodyne HDL-64E S2 for high accuracy mobile scanning," Remote Sensing, vol. 2, no. 6, pp. 1610-1624, jun 2010.

[36] M. Wang, W.-Q. Liu, Y.-H. Lu, X.-S. Zhao, B.-C. Song, Y.-J. Zhang, Y.-P. Wang, C.-H. Lian, J. Chen, Y. Cheng, J.-G. Liu, and Q.-N. Wei, "Study on the measurement of the atmospheric extinction of fog and rain by forward-scattering near infrared spectroscopy," Guang Pu Xue Yu Guang Pu Fen Xi/Spectroscopy Spectral Anal., vol. 28, no. 8, pp. 1776-1780, aug 2008.

[37] T. G. Phillips, N. Guenther, and P. R. McAree, "When the Dust Settles: The Four Behaviors of LiDAR in the Presence of Fine Airborne Particulates," J. Field Robotics, vol. 34, no. 5, pp. 985-1009, aug 2017.

[38] D. Nordin, "Optical Frequency Modulated Continuous Wave (FMCW) Range and Velocity Measurements," Thesis, p. 110, 2004.

[39] C. V. Poulton, D. B. Cole, A. Yaacobi, and M. R. Watts, "Frequency-modulated Continuous-wave LIDAR Module in Silicon Photonics," in Optical Fiber Communication Conference, no. c, Anaheim, 2016, pp. 4-6.

[40] P. Olivier, LEDDAR optical Time-of-Flight sensing technology: A new approach to detection and ranging LeddarTech, Quebec, Tech. Rep. 2016. https://leddartech.com/app/uploads/dlm $\{\backslash\}$ uploads/2016/02 Leddar-Optical-Time-of-Flight-Sensing-Technology-1.pdf

[41] P. F. Mcmanamon, T. A. Dorschner, D. L. Corkum, L. J. Friedman, D. S Hobbs, M. Holz, S. Liberman, H. O. Nguyen, D. P. Resler, R. C. Sharp, E. A. Watson, T. A. Dorschner, L. J. Friedman, D. S. Hobbs, M. Holz, D. P. Resler, and R. C. Sharp, "Optical phased array technology," Proc. IEEE, vol. 84, no. 2, pp. 268-298, feb 1996.

[42] L. Eldada, LiDAR and the Autonomous Vehicle Revolution for Truck and Ride Sharing Fleets San Francisco, 2017. http://www.automated vehiclessymposium.org/avs2018/2017-highlights/2017proceedings

[43] B. Schoettle, Sensor Fusion: A Comparison of Sensing Capabilities of Human Drivers and Highly Automated Vehicles University of Michigan, Tech. Rep. SWT-2017-12, 2017. http://www.umich.edu/ \{ \}umtriswt.

[44] C. Nowakowski, S. E. Shladover, C.-Y. Chan, and H.-S. Tan, "Development of California Regulations to Govern Testing and Operation of Automated Driving Systems," Transportation Res. Record: J. Transpor tation Res. Board, vol. 2489, no. 1, pp. 137-144, 2015.

[45] NHTSA, Federal Automated Vehicles Policy NHTSA, Tech. Rep. September, 2016. https://www.transportation.gov/AV\{\\%\}0Apapers3:// publication/uuid/2E47DA05-79E3-4DA5-BE3B-B172900149A1

[46] Waymo, On the Road to Fully Self-Driving Waymo, Tech. Rep., 2017. https://storage.googleapis.com/sdc-prod/v1/safety-report/ waymo-safety-report-2017-10.pdf

[47] C. Liu, S. Li, F. Chang, and Y. Wang, "Machine vision based traffic sign detection methods: Review, analyses and perspectives," IEEE Access, vol. 7, pp. 86578-86596, 2019.

[48] D. Frejlichowski, "Application of the polar-fourier greyscale descriptor to the automatic traffic sign recognition," in International Conference Image Analysis and Recognition. Springer, 2015, pp. 506-513.

[49] J. Gao, Y. Fang, and X. Li, "Learning local histogram representation for efficient traffic sign recognition," in Image and Signal Processing (CISP), 2015 8th International Congress on. IEEE, 2015, pp. 631-635. 
[50] J. Zhang, M. Huang, X. Jin, and X. Li, "A real-time chinese traffic sign detection algorithm based on modified yolov2," Algorithms, vol. 10, no. 4, pp. 127, 2017.

[51] G. Villalón-Sepúlveda, M. Torres-Torriti, and M. Flores-Calero, "Traffic sign detection system for locating road intersections and roundabouts: the chilean case," Sensors, vol. 17, no. 6, pp. 1207, 2017.

[52] S. Hosseinyalamdary and A. Yilmaz, "A bayesian approach to traffic light detection and mapping," ISPRS J. photogrammetry remote sensing, vol. 125, pp. 184-192, 2017.

[53] Y. Gu, T. Yendo, M. P. Tehrani, T. Fujii, and M. Tanimoto, "Traffic sign detection in dual-focal active camera system," in Intelligent Vehicles Symposium (IV), 2011 IEEE. IEEE, 2011, pp. 1054-1059.

[54] S. Miyata, "Automatic recognition of speed limits on speed-limit signs by using machine learning," J. Imaging, vol. 3, no. 3, pp. 25, 2017.

[55] Y. Yang, H. Luo, H. Xu, and F. Wu, "Towards real-time traffic sign detection and classification,” IEEE Trans. Intell. Transp. Syst., vol. 17, no. 7, pp. 2022-2031, 2016

[56] S. B. Wali, M. A. Hannan, A. Hussain, and S. A. Samad, "An automatic traffic sign detection and recognition system based on colour segmentation, shape matching, and svm," Math. Problems Engineering, vol. $2015,2015$.

[57] R. Timofte, K. Zimmermann, and L. Van Gool, "Multi-view traffic sign detection, recognition, and 3d localisation," Mach. Vis. Appl., vol. 25, no. 3, pp. 633-647, 2014 .

[58] S. Gargoum, K. El-Basyouny, J. Sabbagh, and K. Froese, "Automated highway sign extraction using lidar data," Transportation Res. Record: J. Transportation Res. Board, vol. 2643, no. 1, pp. 1-8, 2017.

[59] S. Weng, J. Li, Y. Chen, and C. Wang, "Road traffic sign detection and classification from mobile lidar point clouds," in 2nd ISPRS International Conference on Computer Vision in Remote Sensing (CVRS 2015), vol. 9901. International Society for Optics and Photonics, 2016, p. 99010A,

[60] L. Zhou and Z. Deng, "Lidar and vision-based real-time traffic sign detection and recognition algorithm for intelligent vehicle," in Intelligent Transportation Systems (ITSC), 2014 IEEE 17th International Conference on. IEEE, 2014, pp. 578-583.

[61] H. Guan, W. Yan, Y. Yu, L. Zhong, and D. Li, "Robust traffic-sign detection and classification using mobile lidar data with digital images," IEEE Journal of Selected Topics in Applied Earth Observations and Remote Sensing, vol. 11, no. 5, 2018.

[62] A. B. Hillel, R. Lerner, D. Levi, and G. Raz, "Recent progress in road and lane detection: a survey," Mach.Vis. Appl., vol. 25, no. 3, pp. 727-745, 2014.

[63] H. Lee, S. Kim, S. Park, Y. Jeong, H. Lee, and K. Yi, "Avm/lidar sensor based lane marking detection method for automated driving on complex urban roads," in Intelligent Vehicles Symposium (IV), 2017 IEEE. IEEE, 2017, pp. 1434-1439.

[64] C.-H. Kum, D.-C. Cho, M.-S. Ra, and W.-Y. Kim, "Lane detection system with around view monitoring for intelligent vehicle," in SoC Design Conference (ISOCC), 2013 International. IEEE, 2013, pp. 215-218.

[65] S.-S. Ieng and D. Gruyer, "Merging lateral cameras information with proprioceptive sensors in vehicle location gives centimetric precision," in Proceedings of the 18th International Technical Conference on the Enhanced Safety of Vehicles. Nagoya, Japan: National Highway Traffic Safety Administration, may 2003.

[66] M. Schreiber, C. Knöppel, and U. Franke, "Laneloc: Lane marking based localization using highly accurate maps," in Intelligent Vehicles Symposium (IV), 2013 IEEE. IEEE, 2013, pp. 449-454.

[67] U. Ozgunalp, R. Fan, X. Ai, and N. Dahnoun, "Multiple lane detection algorithm based on novel dense vanishing point estimation," IEEE Trans. Intell. Transp. Syst., vol. 18, no. 3, pp. 621-632, 2017.

[68] S. Pengpeng, Z. Xiangmo, W. Runmin, M. Haigen, et al. "A 3 d lidar data based dedicated road boundary detection algorithm for autonomous vehicles," IEEE Access, 2019.

[69] B. Yang, L. Fang, Q. Li, and J. Li, "Automated extraction of road markings from mobile lidar point clouds," Photogrammetric Engineering Remote Sensing, vol. 78, no. 4, pp. 331-338, 2012.

[70] T. Li and D. Zhidong, "A new 3d lidar-based lane markings recognition approach," in Robotics and Biomimetics (ROBIO), 2013 IEEE International Conference on. IEEE, 2013, pp. 2197-2202.

[71] H. Zhang, "Rapid Inspection of Pavement Markings Using Mobile Laser Scanning Point Clouds," Ph.D. dissertation, University of Waterloo, mar 2016. https://uwspace.uwaterloo.ca/handle/10012/10343

[72] Y. Nie, O. Chen, T. Chen, Z. Sun, and B. Dai, "Camera and lidar fusion for road intersection detection," in Electrical \& Electronics Engineering (EEESYM), 2012 IEEE Symposium on. IEEE, 2012, pp. 273-276.

[73] D. Kim, T. Chung, and K. Yi, "Lane map building and localization for automated driving using 2 d laser rangefinder," in Intelligent Vehicles Symposium (IV), 2015 IEEE. IEEE, 2015, pp. 680-685.

[74] K. Kaliyaperumal, S. Lakshmanan, and K. Kluge, "An algorithm for detecting roads and obstacles in radar images," IEEE Trans. Veh. Technol., vol. 50, no. 1, pp. 170-182, 2001.
[75] B. Ma, S. Lakshmanan, and A. O. Hero, "Simultaneous detection of lane and pavement boundaries using model-based multisensor fusion," IEEE Trans. Intell. Transp. Syst., vol. 1, no. 3, pp. 135-147, 2000.

[76] F. Janda, S. Pangerl, E. Lang, and E. Fuchs, "Road boundary detection for run-off road prevention based on the fusion of video and radar," in IEEE Intelligent Vehicles Symposium, Proceedings. IEEE, jun 2013, pp. 1173-1178.

[77] A. Mukhtar, L. Xia, and T. B. Tang, "Vehicle detection techniques for collision avoidance systems: A review," IEEE Trans. Intelligent Transportation Systems, vol. 16, no. 5, pp. 2318-2338, 2015.

[78] W.-C. Chang and C.-W. Cho, "Real-time side vehicle tracking using parts-based boosting," in Systems, Man and Cybernetics, 2008. SMC 2008. IEEE International Conference on. IEEE, 2008, pp. 3370-3375.

[79] J. K. Suhr and H. G. Jung, "Rearview camera-based stixel generation for backing crash prevention," IEEE Transactions on Intelligent Trans portation Systems, 2019.

[80] J. D. Alonso, E. R. Vidal, A. Rotter, and M. Muhlenberg, "Lane-change decision aid system based on motion-driven vehicle tracking," IEEE Trans. Veh. Technol., vol. 57, no. 5, pp. 2736-2746, 2008.

[81] K.-T. Song and H.-Y. Chen, "Lateral driving assistance using optical flow and scene analysis," in Intelligent Vehicles Symposium, 2007 IEEE. IEEE, 2007, pp. 624-629.

[82] N. Blanc, B. Steux, and T. Hinz, "Larasidecam: A fast and robust visionbased blindspot detection system," in Intelligent Vehicles Symposium, 2007 IEEE. IEEE, 2007, pp. 480-485.

[83] T. Gandhi and M. M. Trivedi, "Vehicle surround capture: Survey of techniques and a novel omni-video-based approach for dynamic panoramic surround maps," IEEE Trans. Intell. Transp. Syst., vol. 7, no. 3 pp. 293-308, 2006.

[84] N. Bernini, M. Bertozzi, L. Castangia, M. Patander, and M. Sabbatelli, "Real-time obstacle detection using stereo vision for autonomous ground vehicles: A survey," in Intelligent Transportation Systems (ITSC), 2014 IEEE 17th International Conference on. IEEE, 2014, pp. $873-878$.

[85] D. Olmeda, C. Premebida, U. Nunes, J. M. Armingol, and A. de la Escalera, "Pedestrian detection in far infrared images," Integrated Computer-Aided Engineering, vol. 20, no. 4, pp. 347-360, 2013.

[86] S. J. Krotosky and M. M. Trivedi, "On color-, infrared-, and multimodal-stereo approaches to pedestrian detection," IEEE Trans. Intell. Transp. Syst., vol. 8, no. 4, pp. 619-629, 2007.

[87] S. Sivaraman and M. M. Trivedi, "Looking at vehicles on the road: A survey of vision-based vehicle detection, tracking, and behavior analysis," IEEE Trans. Intell. Transp. Syst., vol. 14, no. 4, pp. 1773-1795, 2013.

[88] B. Li, T. Zhang, and T. Xia, "Vehicle detection from 3d lidar using fully convolutional network," arXiv preprint arXiv:1608.07916, 2016.

[89] C. Blanc, R. Aufrere, L. Malaterre, J. Gallice, and J. Alizon, "Obstacle detection and tracking by millimeter wave radar," IFAC Proc. Volumes, vol. 37, no. 8, pp. 322-327, 2004.

[90] F. Garcia, P. Cerri, A. Broggi, A. de la Escalera, and J. M. Armingol, "Data fusion for overtaking vehicle detection based on radar and optical flow," in Intelligent Vehicles Symposium (IV), 2012 IEEE. IEEE, 2012, pp. 494-499.

[91] D. Göhring, M. Wang, M. Schnürmacher, and T. Ganjineh, "Radar/ lidar sensor fusion for car-following on highways," in Automation, Robotics and Applications (ICARA), 2011 5th International Conference on. IEEE, 2011, pp. 407-412.

[92] G. Alessandretti, A. Broggi, and P. Cerri, "Vehicle and guard rail detection using radar and vision data fusion," IEEE Trans. Intell. Transp. Syst., vol. 8, no. 1, pp. 95-105, 2007.

[93] C. Premebida, G. Monteiro, U. Nunes, and P. Peixoto, "A Lidar and vision-based approach for pedestrian and vehicle detection and tracking," in IEEE Conference on Intelligent Transportation Systems, Pro ceedings, ITSC. IEEE, sep 2007, pp. 1044-1049.

[94] Chunsheng, S. Liu, F. Li, Y. Chang, and Wang, "Camera and lidar fusion for on-road vehicle tracking withreinforcement learning," in 2019 IEEE Intelligent Vehicles Symposium (IV), 2019.

[95] E. Dickmanns and A. Zapp, "Autonomous High Speed Road Vehicle Guidance by Computer Vision 1," IFAC Proc. Volumes, vol. 20, no. 5, pp. 221-226, jul 1987.

[96] R. Gregor, M. Lützeler, M. Pellkofer, K. H. Siedersberger, and E. D. Dickmanns, "EMS-Vision: A Perceptual System for Autonomous Vehicles," IEEE Trans. Intell. Transp. Syst., vol. 3, no. 1, pp. 48-59, mar 2002.

[97] A. Broggi, M. Bertozzi, A. Fascioli, and G. Conte, Automatic Vehicle Guidance: the Experience of the ARGO Autonomous Vehicle. World Scientific, 1999.

[98] M. Parent and P. Daviet, "Automatic Driving For Small Public Urban Vehicles," in Proceedings of the Intelligent Vehicles '93 Symposium. IEEE, 1993, pp. 402-407.

[99] U. Ozguner, T. Acarman, and K. Redmill, Autonomous Ground Vehi cles. Artech House, 2007. 
[100] C. Thorpe, T. Jochem, and D. Pomerleau, "The 1997 automated highway free agent demonstration," in Proceedings of Conference on Intelligent Transportation Systems. IEEE, 1997, pp. 496-501.

[101] S. Thrun, M. Montemerlo, H. Dahlkamp, and A. Et, "The robot that won the DARPA Grand Challenge," J. Field Robotics, vol. 23-9, no. 9, pp. 661-692, 2006.

[102] Tartan Racing, Boss at a glance 2005. http://www.tartanracing .org/press/boss-glance.pdf

[103] C. Urmson, DARPA Urban Challenge Final Report for Tartan Racing Tech. Rep., 2007. https://pdfs.semanticscholar.org/3116/38e299acef3 cbd3423649b77ef73c2a94fc1.pdf

[104] M. Montemerlo, J. Becker, S. Bhat, H. Dahlkamp, D. Dolgov, S. Ettinger, D. Haehnel, T. Hilden, G. Hoffmann, B. Huhnke, D. Johnston, S. Klumpp, D. Langer, A. Levandowski, J. Levinson, J. Marcil, D. Orenstein, J. Paefgen, I. Penny, A. Petrovskaya, M. Pflueger, G. Stanek, D. Stavens, A. Vogt, and S. Thrun, "Junior: the Stanford entry in the Urban Challenge,” J. Field Robotics, vol. 25, no. 9, pp. 569-597, sep 2008.

[105] J. Levinson, J. Askeland, J. Becker, J. Dolson, D. Held, S. Kammel, J. Z. Kolter, D. Langer, O. Pink, V. Pratt, M. Sokolsky, G. Stanek, D. Stavens, A. Teichman, M. Werling, and S. Thrun, "Towards fully autonomous driving: Systems and algorithms," in IEEE Intelligent Vehicles Symposium, Proceedings. IEEE, jun 2011, pp. 163-168.

[106] L. Chapell, The Big Bang of autonomous driving 2016. http://www .autonews.com/article/20161219/0EM06/312199908/the-big-bang -of-autonomous-driving

[107] M. Bertozzi, A. Broggi, E. Cardarelli, R. I. Fedriga, L. Mazzei, and P. P. Porta, "VIAC expedition toward autonomous mobility," in IEEE Robotics and Automation Magazine, vol. 18, no. 3, pp. 120-124, sep 2011.

[108] A. Broggi, P. Medici, P. Zani, A. Coati, and M. Panciroli, "Autonomous vehicles control in the VisLab Intercontinental Autonomous Challenge," Annu. Reviews Control, vol. 36, no. 1, pp. 161-171, apr 2012.

[109] A. Broggi, S. Cattani, P. Medici, and P. Zani, "Applications of computer vision to vehicles: An extreme test," Stud. Computational Intell., vol. 411, pp. 215-250, 2013.

[110] J. Levinson, "Automatic Laser Calibration, Mapping, and Localization for Autonomous Vehicles," Ph.D. dissertation, Stanford University, 2011. https://searchworks.stanford.edu/view/9275866

[111] S. J. Anderson, S. B. Karumanchi, K. Iagnemma, and J. M. Walker, "The intelligent copilot: A constraint-based approach to sharedadaptive control of ground vehicles," IEEE Intell. Transp. Syst. Mag., vol. 5 , no. 2 , pp. 45-54, 2013 .

[112] M. Aeberhard, S. Rauch, M. Bahram, G. Tanzmeister, J. Thomas, Y. Pilat, F. Homm, W. Huber, and N. Kaempchen, "Experience, results and lessons learned from automated driving on Germany's highways," IEEE Intell. Transp. Syst. Mag., vol. 7, no. 1, pp. 42-57, 2015.

[113] J. Ziegler, P. Bender, M. Schreiber, H. Lategahn, T. Strauss, C. Stiller, T. Dang, U. Franke, N. Appenrodt, C. G. Keller, E. Kaus, R. G. Herrtwich, C. Rabe, D. Pfeiffer, F. Lindner, F. Stein, F. Erbs, M. Enzweiler, C. Knoppel, J. Hipp, M. Haueis, M. Trepte, C. Brenk, A. Tamke, M. Ghanaat, M. Braun, A. Joos, H. Fritz, H. Mock, M. Hein, and E. Zeeb,
"Making bertha drive-an autonomous journey on a historic route," IEEE Intell. Transp. Syst. Mag., vol. 6, no. 2, pp. 8-20, 2014.

[114] S. Edelstein, Intel/Mobileye Self-Driving Cars Begin Testing in Jerusalem - The Drive 2018. http://www.thedrive.com/tech/20919/intel -mobileye-self-driving-cars-begin-testing-in-jerusalem

[115] S. Scheer, Exclusive: Intel's Mobileye gets self-driving tech deal for 8 million cars 2018. https://www.reuters.com/article/us-israel-tech -intel-mobileye-exclusive/exclusive-intels-mobileye-gets-self -driving-tech-deal-for-8-million-cars-idUSKCN1II0K7

[116] A. Hawkins, Tesla has been working on a backup plan in case its selfdriving promises fail 2017. https://www.theverge.com/2017/8/9/ 16119746/tesla-self-driving-hardware-upgrade-hw-2-5

[117] Ambarella, Ambarella Introduces CV2 4K Computer Vision SoC with CVflow Architecture and Stereovision 2018. https://www.ambarella .com/news/122/74/Ambarella-Introduces-CV2-4K-Computer -Vision-SoC-with-CVflow-Architecture-and-Stereovision

[118] AUVSI, Ambarella testing its fully autonomous EVA vehicle on the roads of Silicon Valley - 2018. https://www.auvsi.org/industry -news/ambarella-testing-its-fully-autonomous-eva-vehicle-roads -silicon-valley

[119] W. team, Introducing Waymo's suite of custom-built, self-driving hardware 2017. https://medium.com/waymo/introducing -waymos-suite-of-custom-built-self-driving-hardware-c47d1714563

[120] L. Zhang, L. Yang, and T. Luo, "Unified saliency detection model us ing color and texture features," PLOS ONE, vol. 11, no. 2, pp. e0149328, 2016.

[121] A. Palazzi, D. Abati, S. Calderara, F. Solera, and R. Cucchiara, "Predicting the Driver's Focus of Attention: the DR(eye)VE Project," (pre print) IEEE Transactions on Pattern Analysis and Machine Intelligence, may 2018.

[122] P. Duthon, M. Colomb, and L. J. Kuntzmann, "Visual saliency on the road: model and database dependent detection," in Actes du 20ème congrès national sur la Reconnaissance des Formes et l'Intelligence Artificielle, jun 2016.

[123] P. M. Bösch, F. Becker, H. Becker, and K. W. Axhausen, "Cost-based analysis of autonomous mobility services," Transport Policy, vol. 64, pp. 76-91, may 2018.

[124] J. Petit, B. Stottelaar, M. Feiri, and F. Kargl, "Remote Attacks on Automated Vehicles Sensors: Experiments on Camera and LiDAR," in Black Hat Europe, 2015, pp. 1-13.

[125] J. Pérez, D. Gonzalez, and V. Milanés, "Vehicle Control in ADAS Applications: State of the Art," in Intelligent Transport Systems: Technologies and Applications. Chichester, UK: John Wiley \& Sons, Ltd, oct 2016, pp. 206-219. http://doi.wiley.com/10.1002/9781118894774 .ch11

[126] Frost \& Sullivan, 2009 European Consumers Desirability and Willingness to Pay for Advanced Safety and Driver Assistance Systems Market Research Tech. Rep, 2010. https://store.frost.com/2009 - european-consumers-desirability-and-willingness-to-pay-for -advanced-safety-and-driver-assistance-systems.html
Index Terms-Automated Driving, LiDAR, Radar, Artificial Vision, Perception. 


\section{Exteroception in Automated Driving is related with information in} the surroundings of the vehicle, as opposed to proprioception that is related with the state of the vehicle itself (speed, accelerations, component integrity).

Behavioral competencies in Automated Driving "refers to the ability of an Automated Vehicle to operate in the traffic conditions that it will regularly encounter" 